\section{Enrique Salazar Grosskelwing*} (1) 0000-0002-3628-0783

Posgrado Institucional en Ciencias Agropecuarias y Manejo de Recusrsos Naturales Tropicales. Universidad Autónoma de Yucatán. Carretera Mérida-Xmatkuil Km 15.5, Mérida, Yucatán, México.

* Autor para correspondencia: Tel: (235) 3234264

Correo electrónico: enriquesalazargross@gmail.com

\title{
Tres casos de infección aguda por Trypanosoma cruzi en perros de Misantla, Veracruz, México
}

\section{Resumen}

Descripción de los casos. Se exponen tres casos clínico-patológicos de tripanosomiasis americana aguda en perros cachorros de la misma camada relacionados con el mismo foco de transmisión en Misantla, Veracruz, México.

Hallazgos clínicos. Los perros mostraron signos clínicos respiratorios, gastrointestinales y sistémicos. Abundantes ooquistes de Cystoisospora sp. en heces. Tripomastigotes en sangre fresca. Estructuras parasitarias compatibles con Trypanosoma sp. en frotis sanguíneo.

Tratamiento y evolución. Se inició tratamiento de sostén, sin embargo, no hubo mejoría y los tres casos clínicos concluyeron en deceso. Al primero se le hizo necropsia.

Pruebas de laboratorio. Coproparasitoscopía directa, cuantificación de glucemia, microscopía directa en sangre y frotis sanguíneo teñido. En la necropsia: citología de médula ósea y análisis histopatológico de miocardio.

Relevancia clínica. Se trata del primer informe de tripanosomiasis aguda en perros de la región. Debido a la inespecificidad de los signos, esta enfermedad se incluye dentro de los diagnósticos diferenciales entre las enfermedades infecciosas y parasitarias agudas o crónicas en los perros de la zona. El carácter zoonótico de esta enfermedad vectorial desatendida representa un riesgo para la salud pública y animal, por lo que, deben considerarse medidas de monitoreo, prevención y control que disminuyan la probabilidad de zoonosis.

Palabras clave: enfermedad de Chagas, Misantla, parasitemia, perros, Trypanosoma sp.

Recibido: 2019-11-24

Aceptado: 2020-03-31

Publicado: 2020-05-13

Información y declaraciones adicionales en la página 9

(C) Derechos de autor: Enrique Salazar Grosskelwing 2020

acceso abierto $\boldsymbol{\varnothing}$

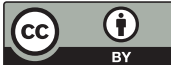

Distribuido bajo una Licencia Creative Commons Atribución 4.0 Internacional (CC-BY 4.0)

\section{Una forma de citar este artículo:}

Salazar Grosskelwing E. Tres casos de infección aguda por Trypanosoma cruzi en perros de Misantla, Veracruz, México. Clínica veterinaria: abordaje diagnóstico y terapéutico. 2020;6:e56202062. doi: 10.22201/fmvz.23958766e.202056. 


\title{
Three cases of acute Trypanosoma cruzi infection in dogs from Misantla, Veracruz, Mexico
}

\author{
Abstract \\ Description of the cases. Three clinical-pathological cases of acute American \\ trypanosomiasis in puppies from the same litter related to the same focus \\ of transmission in Misantla, Veracruz, Mexico are exposed. \\ Clinical findings. The dogs showed clinical respiratory, gastrointestinal, and \\ systemic signs. Abundant oocysts of Cystoisospora sp. in feces. Trypomasti- \\ gotes in fresh blood. Parasitic structures compatible with Trypanosoma sp. \\ in blood smear. \\ Treatment and evolution. Supportive treatment was started, however, there \\ was no improvement and all clinical cases ended in death. The necropsy \\ was performed in the first case. \\ Lab tests. Direct coproparasitoscopy, blood glucose quantification, direct blood \\ microscopy and stained blood smear. In necropsy: bone marrow cytology \\ and histopathological analysis of myocardium. \\ Clinical relevance. This is the first report of acute trypanosomiasis in dogs in \\ the region. Due to the non-specificity of the signs, this disease is included \\ in the differential diagnoses of acute or chronic infectious and parasitic dis- \\ eases in the dogs in the area. The zoonotic nature of this neglected vec- \\ tor-borne disease represents a risk to public and animal health, therefore, \\ monitoring prevention and control measures that decrease the probability \\ of zoonosis should be considered.
}

Keywords: Chagas disease, dogs, Misantla, parasitemia, Trypanosoma sp. 


\section{Descripción de los casos}

Una cachorra mestiza de dos meses de edad proveniente de las orillas de Misantla, Veracruz, México, se examinó por signología respiratoria y gastrointestinal en una clínica privada del lugar en septiembre de 2019. Ocho y nueve días después, también se examinaron a dos de sus hermanos de la misma edad por un cuadro similar.

\section{Hallazgos clínicos e interpretación}

El responsable del animal notó inicialmente que la perra presentaba hematoquecia y vómito, mientras sus hermanos no presentaban signos aparentes de enfermedad. La paciente estaba estuporosa, tenía taquipnea y postura ortopnéica con marcada dificultad respiratoria. Además, mucosas pálidas y exudado purulento palpebral y nasal.

En el análisis coproparasitoscópico directo se observaron abundantes ooquistes de Cystoisospora sp. La glucosa sanguínea fue de $28 \mathrm{mg} / \mathrm{dL}$. Durante el análisis microscópico de sangre se encontraron abundantes tripomastigotes en movimiento. Los diagnósticos diferenciales incluyeron distemper, parvovirosis, gastroenteritis bacteriana, nematodosis y protozoosis intestinal, bronconeumonía bacteriana, entre otros.

Una semana después, un hermano de la misma camada fue examinado clínicamente porque comenzó con signos similares: patrón respiratorio restrictivo con postura ortopnéica, exudado nasal y palpebral purulento, mucosas pálidas, anorexia y depresión. En sangre fresca, también se hallaron abundantes tripomastigotes en movimiento.

Al día siguiente, el tercer cachorro de la camada también se examinó clínicamente. Estaba alerta con ligero exudado nasal purulento, taquipnea sin postura ortopnéica y buen ánimo. Se analizó su sangre de manera directa en el microscopio óptico: se observaron escasos tripomastigotes. El cachorro había estado comiendo y bebiendo con normalidad, sin embargo, una semana después su estado empeoró de forma súbita. Se le dificultaba respirar y tenía anorexia.

\section{Tratamiento y evolución}

Se instauró fluidoterapia endovenosa con suero glucosado al $10 \%$ en la cría hembra junto con sulfametoxazol-trimetoprima ( $24 \mathrm{mg} / \mathrm{kg}$ de la combinación con proporción $5: 1$, respectivamente), metronidazol (15 mg/kg, Iv) y citrato de maropitant ( $1 \mathrm{mg} / \mathrm{kg}, \mathrm{sc}$ ); pero, por la severidad de los signos, el responsable del animal decidió la eutanasia, después de la cual se hizo la necropsia (Figuras 1 y 2). En los hermanos se usó una combinación de antihelmínticos por vía oral en una dosis como desparasitación preventiva: ivermectina $(0.006 \mathrm{mg} / \mathrm{kg})$, pomoato de pirantel (14.4 mg/kg), febantel $(15 \mathrm{mg} / \mathrm{kg})$ y prazicuantel $(5 \mathrm{mg} / \mathrm{kg})$. Una semana después, el responsable, una vez más, optó por la eutanasia para el segundo paciente de la camada. El tercer cachorro, luego de tres días de que comenzó con el cuadro respiratorio, murió. 


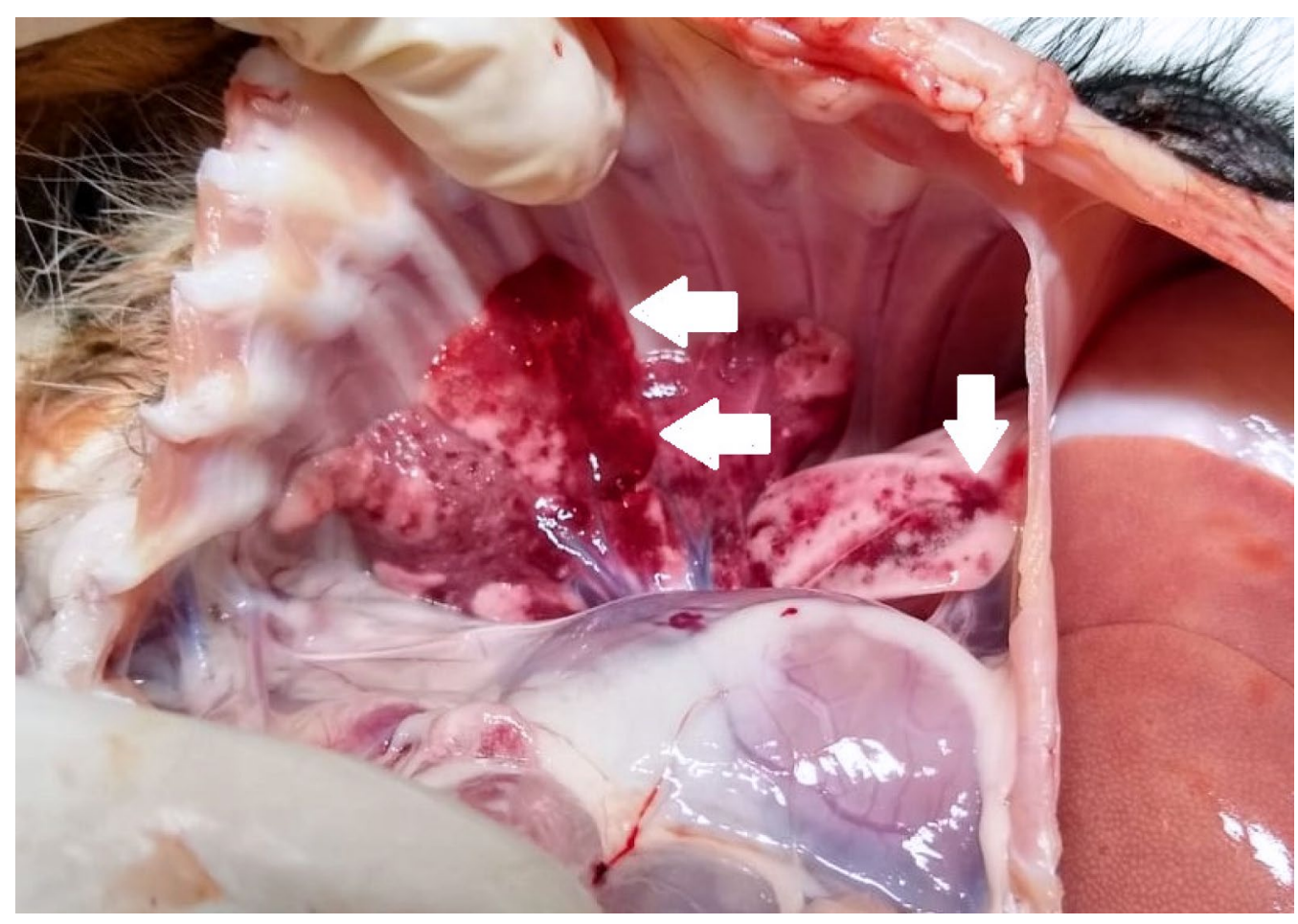

Figura 1. Necropsia. Pulmón con lesiones hemorrágicas difusas (flechas).

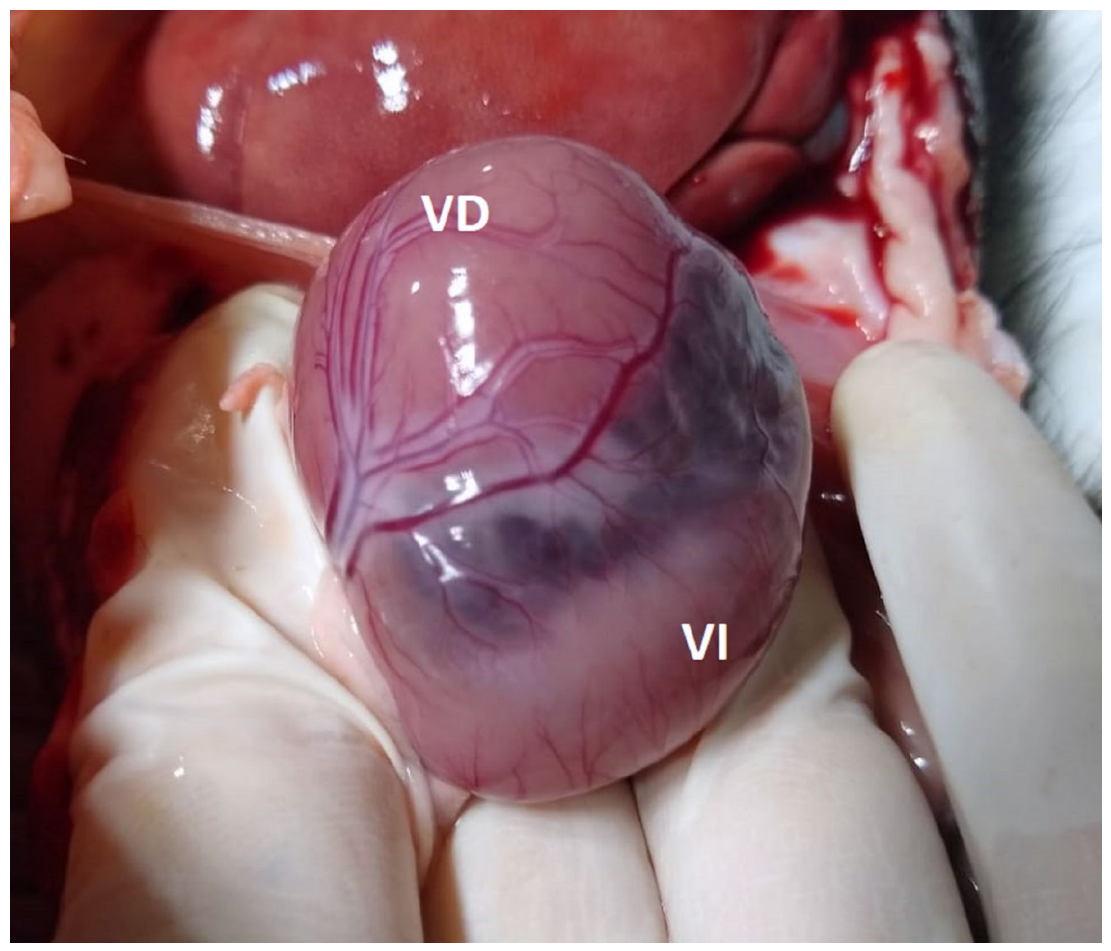

Figura 2. Necropsia. Corazón con apariencia sugerente de agrandamiento del ventrículo derecho (VD) en comparación con el ventrículo izquierdo (VI). 


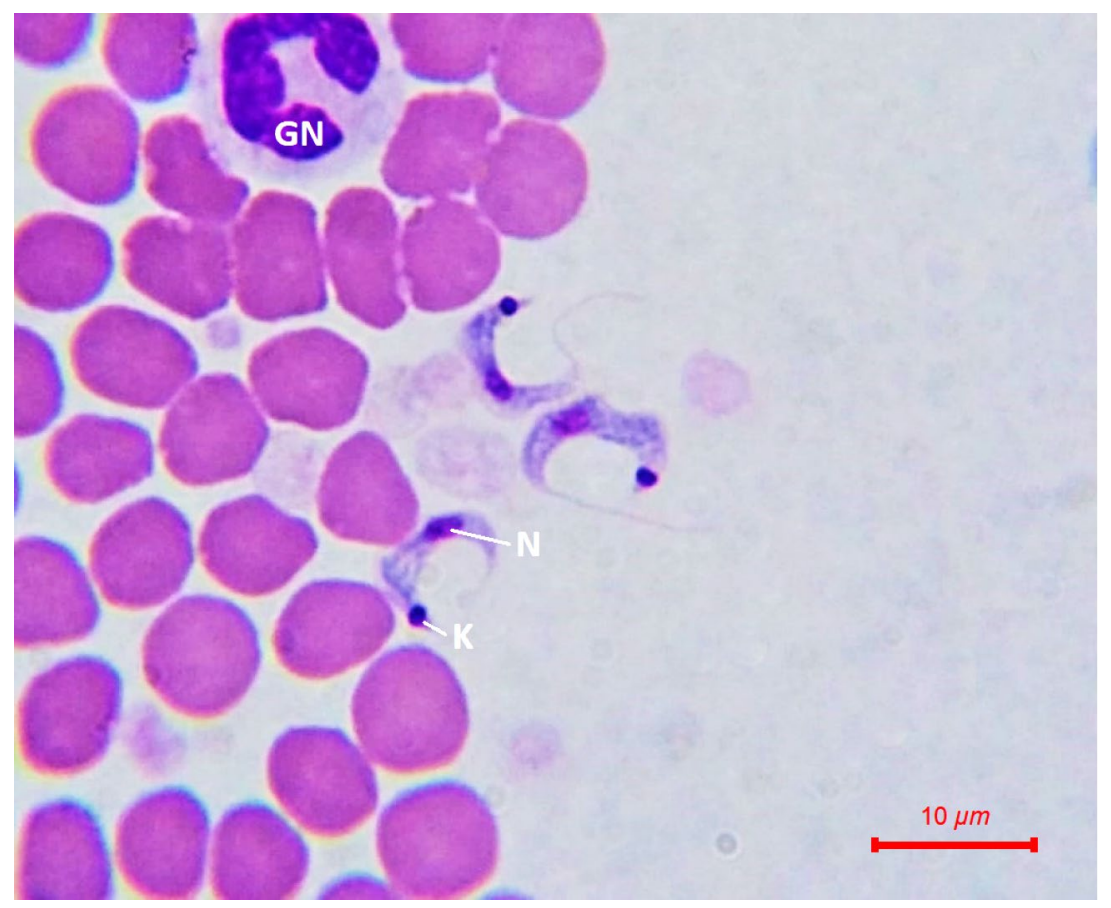

Figura 3. Tres tripomastigotes en frotis sanguíneo. K: kinetoplasto. N: núcleo. GN: granulocito neutrófilo. Tinción Diff-Quick. Aumento 1000X.

\section{Pruebas de laboratorio}

Se realizó un análisis coproparasitoscópico directo a partir de una muestra de heces de un hisopado rectal. Análisis directo al microscopio óptico, de sangre fresca colectada en un tubo Microtainer con K2 EDTA (Franklin Lakes, NJ), y cuantificación de glucemia mediante OneTouch UltraMini de Johnson \& Johnson Medical Devices (Miami, FL). También se realizó un frotis sanguíneo teñido con Diff-Quick (Miami, FL) para su observación microscópica.

En la primera cría los tripomastigotes en sangre se confirmaron con frotis sanguíneo (Figura 3). En la necropsia de este caso, se tomó una muestra citológica de médula ósea de fémur, que se preparó bajo la misma metodología del frotis sanguíneo, y una muestra de miocardio que se remitió al servicio de patología. En la citología de médula ósea se observaron algunos monocitos con escasos amastigotes (Figura 4). Mientras el análisis histopatológico del miocardio reveló un infiltrado inflamatorio linfocítico y pseudoquistes de amastigotes en ese tejido (Figura 5).

\section{Discusión y relevancia clínica}

Aunque este estudio carece de caracterización molecular del agente, otras evidencias como la morfología del parásito en el frotis sanguíneo, su presencia en tejido muscular cardíaco y el cuadro clínico de los animales, sugieren que se trata de Trypanosoma (Schizotrypanum) cruzi, el protozoo causante de la tripanosomiasis americana o enfermedad de Chagas. ${ }^{1}$ Esta zoonosis afecta especialmente a zonas rurales de América Latina, aunque comienza a cobrar importancia en Estados Unidos de América, Japón y países europeos. ${ }^{2}$ Es una enfermedad de transmisión 


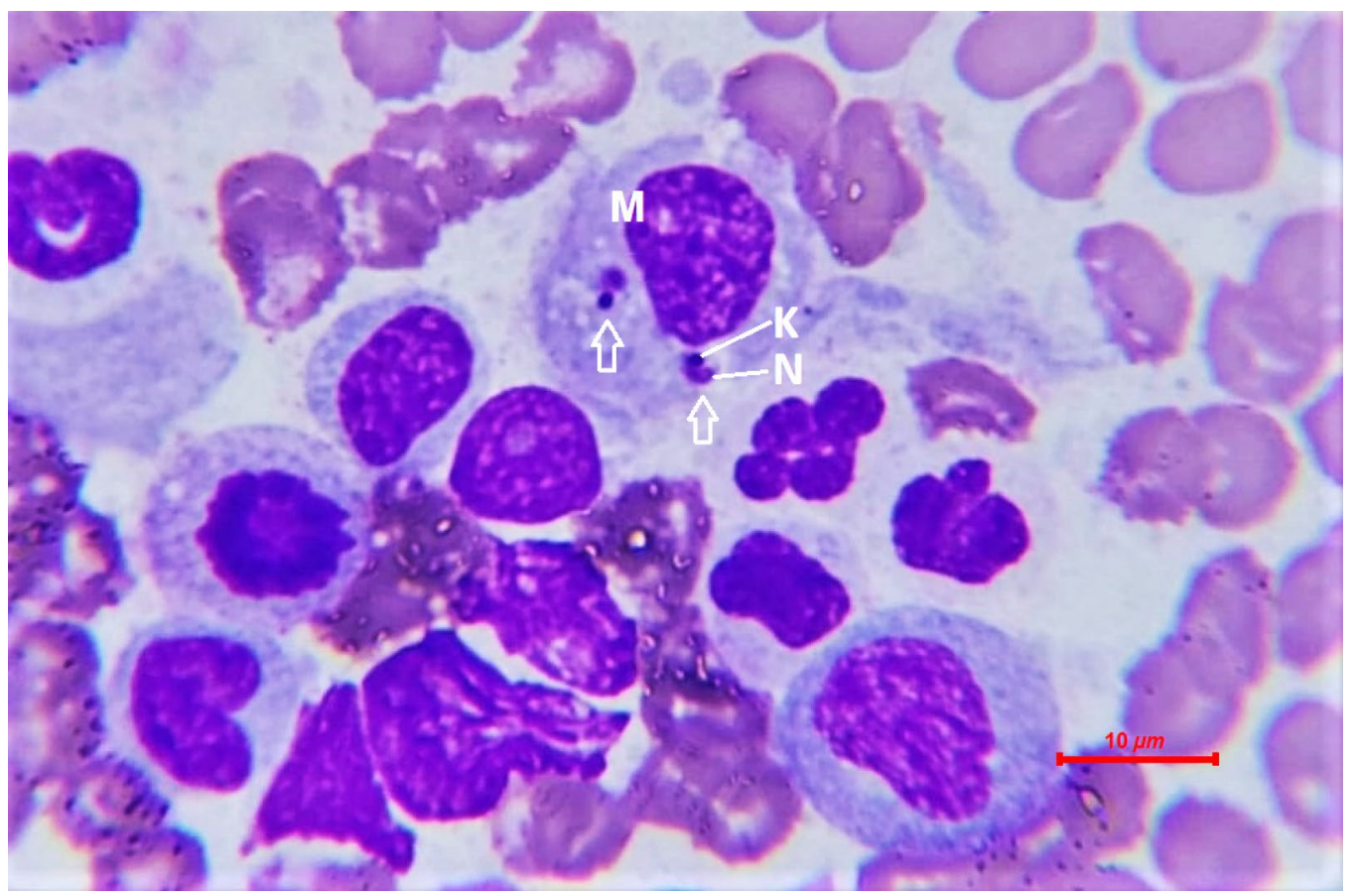

Figura 4. Citología de médula ósea. Se observa un monocito (M) con dos amastigotes en el citoplasma (flechas). Se distingue el núcleo (N) y el kinetoplasto (K) en cada amastigote. Tinción Diff-Quick. Aumento 1000X.

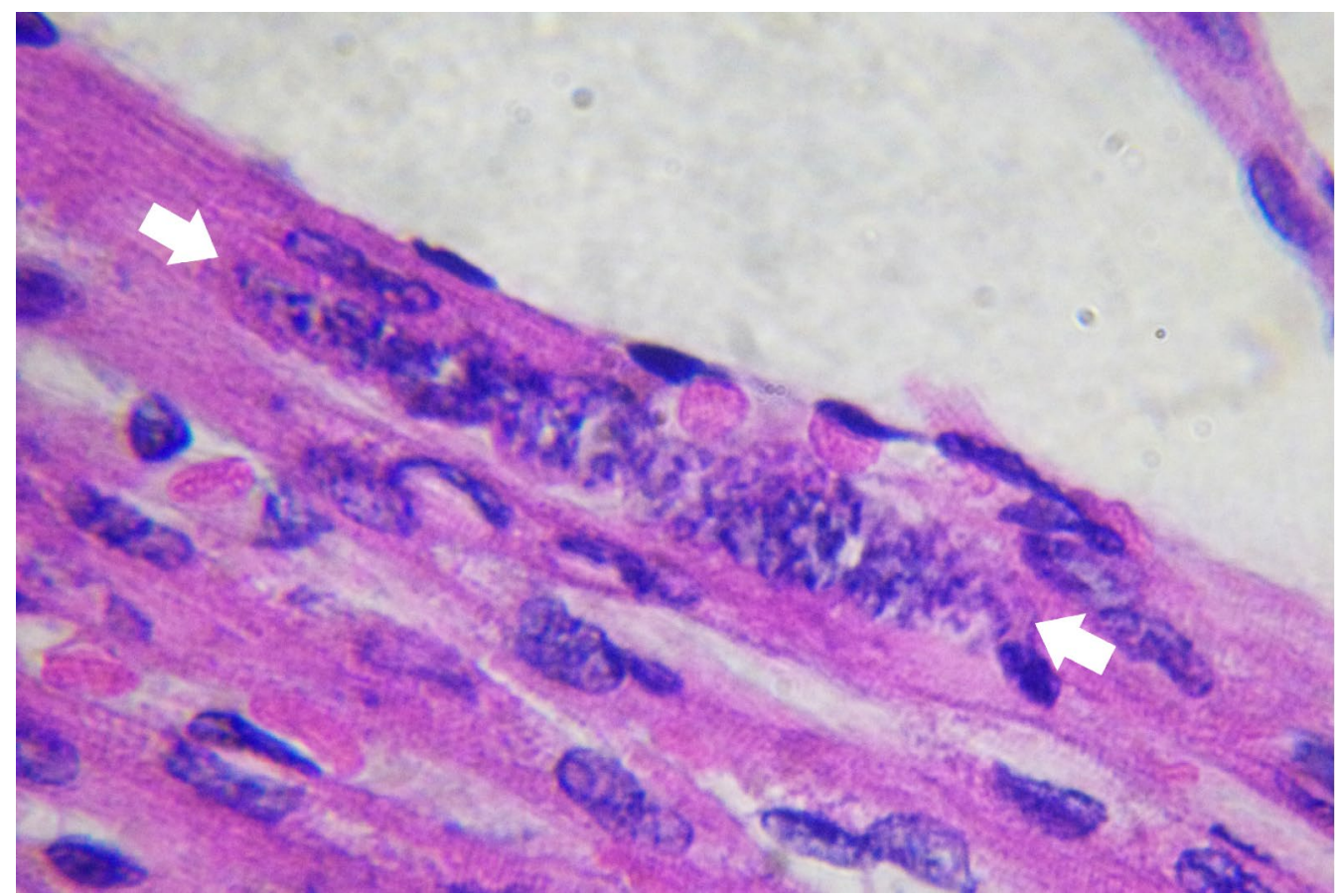

Figura 5. Corte histológico de miocardio. Se observan estructuras parasitarias en las fibras musculares (flechas). Tinción H\&E. Aumento 400X. Foto: Dr. Rodolfo Ramos Beltrán. 
vectorial donde participan chinches hematófagas de la familia Triatominae, los vectores en el continente americano pertenecen a los géneros Triatoma, Rodnius y Panstrongylus. ${ }^{3}$

En México, dos tercios del territorio se consideran zonas de riesgo de transmisión, se calcula que alrededor de un millón de habitantes padecen la infección y cerca de 30 millones están en riesgo de contraerla. ${ }^{4}$ Veracruz pertenece a la zona de riesgo de transmisión. En un análisis serológico realizado en este estado de la República Mexicana, las regiones que presentaron mayores tasas de infección en humanos fueron Pánuco, Tuxpan, Xalapa y San Andrés Tuxtla. ${ }^{5}$

No hay muchos datos sobre la epidemiología de la enfermedad en los perros de la región. Ruiz-Aguilar ${ }^{6}$ reportó una seroprevalencia de $9.4 \%$ mediante la prueba de inmunofluorescencia indirecta para la detección de anticuerpos anti-T. cruzi en 340 perros del municipio de La Antigua, Veracruz, a 160 km del sitio del presente estudio.

Por otro lado, el complejo ciclo de transmisión del protozoo involucra a mamíferos domésticos, silvestres y sinantrópicos. ${ }^{7}$ Las especies de mamíferos que participan como reservorio de parásitos tripanosomátidos como Didelphis virginiana, Didelphis marsupialis y Dasypus novemcinctus son endémicos en la región. ${ }^{8,2}$ De la misma forma, en la zona se ha reportado Triatoma dimidiata, el principal triatomino transmisor de la enfermedad de Chagas en el Golfo de México. ${ }^{9}$

El perro doméstico participa en este ciclo como centinela de la infección, está predispuesto a enfermar y transmitir el parásito nuevamente al vector. La signología de los perros enfermos en este trabajo fue similar a la descrita por Kjos et al. ${ }^{10}$ Entre los signos clínicos descritos se encuentran letargia, anorexia, dificultad respiratoria y anemia. Además, observaron que la mayoría de las muertes por enfermedad aguda ocurría en animales menores de un año.

Por otro lado, Eloy y Lucheis $^{3}$ mencionan que la hipoglucemia es una anormalidad de laboratorio típica en infecciones naturales, pero un hallazgo que no se observa en infecciones experimentales. En perros, la mortalidad es más alta en animales menores de seis meses, que en perros mayores. ${ }^{11}$

La vía de transmisión del parásito no se da solo a través de la contaminación de heridas o abrasiones cutáneas con heces de triatomino infectado, también puede resultar por la ingestión de triatominos infectados o alimentos contaminados con sus heces. La transmisión oral es la principal ruta de infección en muchas especies de mamíferos carnívoros silvestres. ${ }^{11}$

Se ha demostrado que la ingesta de triatominos es una forma efectiva y natural de contraer el parásito, en especial si los invertebrados son parte de la dieta del mamífero. ${ }^{12}$ De igual manera, la comida contaminada con heces u orina de zarigüeyas infectadas puede transmitirla. ${ }^{11}$ Por otro lado, se ha descrito la transmisión vertical vía trasplacentaria y a través de la leche materna. ${ }^{11}$

Lo anterior sugiere que no necesariamente los perros estudiados fueron infectados debido a la picadura del vector, también pudo ser a través de la ingestión del triatomino infectado o de sus heces, o por contacto con mamíferos sinantrópicos: reservorio del parásito. Además, la transmisión vertical es otra posibilidad debido a la edad de los cachorros y a que la parasitemia en todos ellos fue en el mismo periodo.

El tratamiento específico para la fase aguda no está disponible comercialmente y los informes de perros tratados son escasos. Los fármacos para el tratamiento 
de la tripanosomiasis americana son benznidazol y nifurtimox, el primero reacciona con menos efectos secundarios. ${ }^{13}$ La dosis del benznidazol es de 5 a $10 \mathrm{mg} / \mathrm{kg}$ cada $24 \mathrm{~h}$, durante dos meses. $Y$ de nifurtimox se administran de 2 a $7 \mathrm{mg} / \mathrm{kg}$ cada 6 h durante tres a cinco meses. Ambos fármacos por vía oral. ${ }^{13}$

Los hallazgos en este informe confirman la presencia del protozoo en los perros domésticos de la ciudad de Misantla. La presencia del agente, el vector y los reservorios en esta región selvática y boscosa generan el ambiente propicio para el cumplimiento del ciclo de transmisión del parásito. Lo anterior sugiere que el centro del estado podría tener una alta prevalencia de infección en perros, lo que expone a las personas y animales domésticos a la enfermedad, en particular si el vector abunda.

Una limitación importante del presente informe sin duda es la demostración del proceso en un número muy reducido de casos. Por esta razón, se requiere un análisis epidemiológico con una muestra representativa de la población canina para estimar la prevalencia de infección. Porfirio et al. ${ }^{7}$ recomiendan realizar estudios transversales mediante la detección de anticuerpos anti-T. cruzi y material genético del agente simultáneamente. Los resultados en ambas pruebas en el estudio de las enfermedades causadas por tripanosomátidos permiten estimar el predominio de las infecciones agudas o crónicas.

La ausencia de casos previos sugiere que esta enfermedad ha sido subdiagnosticada en los perros de esta región. La tripanosomiasis americana o enfermedad de Chagas deberá considerarse dentro de los diagnósticos diferenciales de las enfermedades infecciosas caninas agudas o crónicas en la zona, sobre todo si los animales se encuentran cerca de áreas selváticas o en contacto con reservorios silvestres.

Debido a la importancia de la enfermedad para la salud pública y las intrincadas relaciones del parásito con los animales y el medio ambiente, esta zoonosis debe ser abordada bajo la perspectiva de una sola salud, donde la medicina humana y veterinaria converjan para aportar alternativas de prevención y control. ${ }^{14,15}$ Además, estas medidas tendrán consideraciones específicas sobre la conservación y el cuidado ambiental, pues las alteraciones antropogénicas de los ecosistemas aumentan notoriamente la probabilidad de emergencia y reemergencia de enfermedades zoonóticas. ${ }^{13}$ 


\section{Financiamiento}

El presente trabajo, incluidos los análisis de laboratorio, fueron financiados por el autor.

\section{Agradecimientos}

Al doctor Rodolfo Ramos Beltrán por la elaboración del análisis histopatológico.

\section{Conflictos de interés}

El autor declara no tener conflicto de intereses.

\section{Referencias}

1. Martins F, Texeira W, Barreto G, Carvalho G, Macedo D, Helena J, et al. The reservoir system for Trypanosoma (Kinetoplastida, Trypanosomatidae) species in large neotropical wetland. Acta Trop. 2019;199(2019):1-7. doi: 10.1016/j. actatropica.2019.105098.

2. Bern C, Kjos S, Yabsley MJ, Montgomery SP. Trypanosoma cruzi and Chagas' disease in the United States. Clin Microbiol Rev. 2011;24(4):655-81.

3. Eloy L, Lucheis S. Canine trypanosomiasis: etiology of infection and implications for public health. J Venom Anim Toxins Incl Trop Dis. 2009;15(4):589-611.

4. Salazar-Schettino PM, Bucio-Torres MI, Rojo-Medina J, Ruiz-Matus C, De Alba-Alvarado MC, Castillo-Saldaña DR, et al. Enfermedad de Chagas en México. Gac Med Mex. 2016;59(3):1-16.

5. Segura EL, Escobar-Mesa A. Epidemiología de la enfermedad de Chagas en el estado de Veracruz. Salud Publica Mex. 2005;47(3):201-8.

6. Ruiz-Aguilar ME. Seroprevalencia y factores de riesgo asociados a Trypanosoma cruzi en perros de comunidades rurarles del municipio de La Antigua, Veracruz, México [tesis de maestría]. Veracruz (MX): Universidad Veracruzana; 2015.

7. Porfirio GE, Santos FM, Macedo GC, Barreto WTG, Campos JBV, Meyers AC, et al. Maintenance of Trypanosoma cruzi, T. evansi and Leishmania spp. by domestic dogs and wild mammals in a rural settlement in Brazil-Bolivian border. Int J Parasitol Parasites Wildl. 2018;7(3):398-404.

8. Rodríguez-Macedo M, González-Christen A, León-Paniagua LS. Diversidad de los mamíferos silvestres de Misantla, Veracruz, México. Rev Mex Biodivers. 2014;85(1):262-75.

9. Tay J, Salazar PM, Bucio MI, Zárate R, Zárate L. Estado actual de nuestros conocimientos sobre la enfermedad de Chagas en la Republica Mexicana. Rev la Soc Bras Med Trop Med Trop. 1979;14(1):29-67.

10. Kjos SA, Snowden KF, Craig TM, Lewis B, Ronald N, Olson JK. Distribution and characterization of canine Chagas disease in Texas. Vet Parasitol. 2008; 152(1):249-56.

11. Gürtler RE, Cardinal M V. Reservoir host competence and the role of domestic and commensal hosts in the transmission of Trypanosoma cruzi. Acta Trop. 2015;151(1):32-50. doi: 10.1016/j.actatropica.2015.05.029.

12. Lopes-Rocha F, Rodrigues-Roque AL, Saab de Lima S, Carvalho-Cheida C, Gemesio-Lemos F, Cavalcanti de Azevedo F, et al. Trypanosoma cruzi infection in neotropical wild carnivores (Mammalia:Carnivora): at the top of the T. cruzi transmission chain. PLoS One. 2013;8(7):1-12. 
13. Barr SC. Canine Chagas' disease (american trypanosomiasis) in North America. Vet Clin North Am Small Anim Pract. 2009;39(6):1055-64. doi: 10.1016/j. cvsm.2009.06.004.

14. Waleckx E, Camara-Mejia J, Ramirez-Sierra MJ, Cruz-Chan V, Vazquez-Narvaez $\mathrm{S}$, Najera-Vazquez $\mathrm{R}$, et al. Una intervención innovadora de ecosalud para el control vectorial de la enfermedad de Chagas en Yucatán, México. Rev Biomed. 2015;26(2):75-86.

15. Reyes-Novelo E, Ruíz-Piña H, Escobedo-Ortegón J, Rodríguez-Vivas I, Bolio-González ME, Polanco-Rodríguez M, et al. Situación actual y perspectivas para el estudio de las enfermedades zoonóticas emergentes, reemergentes y olvidadas en la Península de Yucatán, México. Trop Subtrop Agroecosystems. $2011 ; 14: 35-54$. 